\title{
A Parallel Approach to Post Source Decay MALDI-TOF Analysis
}

\author{
D. J. Kenny, J. M. Brown, M. E. Palmer, M. F. Snel, and R. H. Bateman \\ Waters Corporation, Manchester, United Kingdom
}

We present a novel enhancement to matrix-assisted laser desorption ionization (MALDI) post-source decay (PSD) analysis whereby fragment ions from multiple precursor ions are acquired into the same spectrum without employing a timed ion gate to preselect each parent ion. Fragment ions are matched to their corresponding precursor ions by comparing spectra acquired at slightly different reflectron electric fields. By measuring the difference in time-of-flight (TOF) between the two spectra for each fragment, it is possible to calculate the mass of the fragment ion and its parent. This new "parallel PSD" technique reduces analysis time and consumes less sample than conventional PSD, which requires an ion gate for serial preselection of precursor ions. (J Am Soc Mass Spectrom 2006, 17, 60-66) (C) 2005 American Society for Mass Spectrometry

$\mathrm{M}$ ALDI-TOF mass spectrometers equipped with reflectrons are able to analyze fragment ions produced from precursor ions that spontaneously decompose in flight. Such ions are generally referred to as metastable ions, and the process of decomposition in the field free region between the ion source and the reflectron is commonly referred to as post source decay (PSD) [1-3]. The analysis of such PSD ions is an established technique that is capable of providing complementary MS/MS information.

Peptide mass fingerprinting (PMF) is a common approach to the identification of unknown peptides and proteins [4-7]. There are, however, some instances where PMF is unable to identify a particular protein from a tryptic digestion with confidence. This could be due to a number of factors, such as too few tryptic peptides, e.g., from tryptic digestion of low molecular weight proteins, or because there is a mixture of proteins present, resulting in an overly complex mass spectrum. In these situations, it is advantageous to be able to include even short pieces of sequence information to provide added specificity in databank searching $[8,9]$. Furthermore, the acquisition of several PSD spectra matching to sequences in the same protein will then give a high level of confidence in protein identification. There is increasing interest in protein analysis by MALDI of complex mixtures which require prior separation by liquid chromatography, a technique referred to as LC-MALDI. Analogous to analysis of complex mixtures by electrospray (ESI), it is difficult to identify proteins by $\mathrm{PMF}$, and identification requires MS/MS information [10].

Published online December 15, 2005

Address reprint requests to Dr. D. J. Kenny, Waters Corporation, MS Technologies Center, Floats Road, Wythenshawe, Manchester M23 9LZ, United Kingdom. E-mail: daniel_kenny@waters.com
Figure 1 is a schematic of a typical mass spectrometer which is capable of performing a PSD experiment. To obtain a precursor ion spectrum, a pulsed ultra violet (UV) laser is focused onto an analyte/matrix sample. The resulting ions are accelerated out of the ion source and into the field free region. From here, ions are reflected by the reflectron so as to return to the field free region and then impact upon the detector. In a conventional PSD experiment, however, a timed electrostatic ion gate located after the ion source and within the field free region is opened briefly to allow a single precursor mass and its associated fragments to pass through into the reflectron and be analyzed. The ion gate thus ensures that substantially all detected fragment ions are attributed to the correct precursor.

PSD fragment ions are formed within the field free region before entering the reflectron. Therefore, they have approximately the same velocity as their precursor ion and consequently they have a lower kinetic energy that is proportional to their mass. Hence, a fragment ion will penetrate less into the reflectron and will follow a shorter path. As a consequence, the fragment ion will arrive at the detector before its precursor ion. Additionally, as they do not penetrate into the reflectron to the same depth as precursor ions, low mass fragments are not correctly time focused at the detector. This problem may be alleviated by successively reducing the reflectron field in steps and acquiring data for each step. The focused regions of the resultant spectra, or segments, may then be "stitched" together to form a single complete PSD spectrum [3]. An alternative approach is to use a reflectron with a nonlinear electric field such as a quadratic or a harmonic reflectron [11]. These reflectrons allow a broad energy range to be focused and, hence, allows a PSD spectrum to be acquired without the need to step the reflectron field. Such reflectrons, however, have the added disadvantage that the electric 


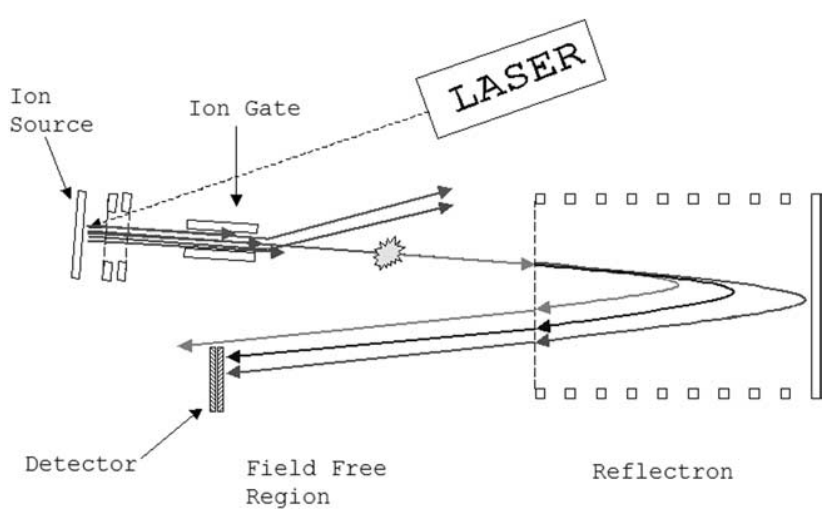

Figure 1. Schematic of a typical experimental setup for a PSD experiment

field is not only nonlinear axially, but also have a field in the radial direction. This leads to increased divergence for off-axis ions, which in turn results in reduced resolution, mass measurement accuracy, and sensitivity [12]. A further approach is utilized in "TOF-TOF" instruments in which the fragment ions are re-accelerated after fragmentation but before their entry into the reflectron. These ions consequently penetrate substantially into the reflectron and allow fragment ion spectra to be acquired without stepping the reflectron voltage. However, such instruments still require the use of an ion gate and therefore still acquire MS/MS data in a serial manner.

In the PSD experiment described above, the timed ion gate is an important and necessary component since, if no ion gate were present, fragment ions from all of the precursor ions would be transmitted and recorded simultaneously. Hence, if the mass spectrum contains more than one precursor ion, it would not be known which fragment ion originated from which precursor ion, and therefore it would not be possible to assign a mass to each fragment ion. This inherent requirement to use an ion gate consequently leads to serial data acquisition and therefore a long acquisition time and increased sample consumption. Furthermore, it is not known which peptides are most likely to fragment by PSD until after they have been analyzed. Hence, a lot of time and sample can be consumed attempting to acquire PSD fragment ion data from the less productive peptide ions. In some cases, all of the sample may be consumed before PSD ions of sufficient abundance have been acquired.

Timed ion gates can have a number of problems associated with them. They can perturb the ion motion of the ions of interest, i.e., transmitted ions may be axially and/or radially accelerated or decelerated by stray electric fields from the ion gate. Fragments of low-energy are particularly vulnerable to the stray electric fields of the device. If the electronic pulse required to gate the ions is too slow or overshoots and oscillates, fragment ion mass resolution and transmission may be affected. The Bradbury Nielson type ion gate [13], which is comprised of parallel wires with voltages of alternating polarity applied to successive wires, will reduce ion transmission even when the gate is set to transmit ions because some ions will strike the wires and become neutralized or scattered. Ions that are deliberately deflected by the ion gate will strike other parts of the mass spectrometer and care must be taken to ensure that stray ions, or secondary ions, do not arrive at the detector. Otherwise, weak fragment ions from minor precursor ions in complex mixtures can be obscured by the presence of stray ions caused by the deflection of the more abundant precursor ions.

Here we describe a method of acquiring multiple PSD spectra in parallel, avoiding the use of a timed ion gate, therefore overcoming some of the problems and limitations present in conventional methods.

\section{Experimental}

All experiments described were performed on a MALDI micro MX mass spectrometer (Waters Corporation, Manchester, UK). Protein digest standards and $\alpha$-cyano-4-hydroxycinnamic acid (CHCA) matrix were both obtained from Waters Corporation. The matrix was used at a concentration of $10 \mathrm{mg} / \mathrm{mL}$ (unless specified otherwise) dissolved in a solution containing 1:1 acetonitrile and ethanol. Standard peptides were obtained from Sigma-Aldrich (Poole, UK). All peptides and protein digests were premixed 1:1 with matrix with $1 \mu \mathrm{L}$ of sample/matrix solution deposited onto the MALDI target plate. The samples were allowed to dry under ambient conditions before being loaded into the mass spectrometer.

\section{Theory}

Our new method requires that the time of flight of the PSD fragment ions are measured twice at two relatively close reflectron field settings. This small difference in the reflectron field is defined as a "minor" step as opposed to the "major" steps which are conventionally used to successively bring lower mass PSD fragments into focus. Whereas a major step might typically be a $25 \%$ reduction in the reflectron field, the minor step is only a $4 \%$ reduction in the reflectron field. As a result of having acquired major and minor spectra, two independent sets of data exist for each major step. This provides sufficient information to enable the calculation of the precursor and fragment ion masses for each observed fragment peak. This procedure is repeated for each PSD segment or "major" step in the conventional way to ensure good energy focusing over the entire fragment ion mass range.

This new method may be better understood by first considering the conventional PSD time of flight equation. The time of flight (TOF) is given by: 


$$
T O F=\sqrt{m_{p}}\left(a+\frac{m_{f}}{m_{p}} \cdot b\right)
$$

Here, $m_{p}$ is the mass of the precursor ion, $m_{f}$ is the mass of its associated PSD fragment ion and $a$ and $b$ are instrumental coefficients dependent upon voltages and dimensions of the system which may be determined via calibration on a known compound. The first term of the equation represents the time of flight in the ion source and the field free region, whereas the second term represents the time of flight in the reflectron of the mass spectrometer. An important point to note is that coefficient $b$ is inversely proportional to the reflectron voltage.

Since the mass (and TOF) of the precursor ion is a known quantity in a conventional PSD experiment (as it is defined by the ion gate timing), the mass of a PSD fragment is easily derived from its time of flight using eq 1 . However, in an experiment with no precursor ion selection, neither the mass of the fragment nor the mass of the precursor is known and so eq 1 cannot be solved.

One solution to this problem is to measure the time of flight twice for each fragment, i.e., to record two spectra, but at slightly different reflectron voltages. This then leads to the two following time of flight equations:

$$
\begin{aligned}
& T O F_{\text {major }}=\sqrt{m_{p}}\left(a+\frac{m_{f}}{m_{p}} \cdot b_{\text {major }}\right), \\
& T O F_{\text {minor }}=\sqrt{m_{p}}\left(a+\frac{m_{f}}{m_{p}} \cdot b_{\text {minor }}\right) .
\end{aligned}
$$

It can be readily seen that if the reflectron voltage is changed in the described manner, the shift in the time of flight $\triangle T O F$ for a particular fragment is proportional to the change $\Delta b$ in coefficient $b$ :

$$
\Delta T O F=\Delta b\left(\frac{m_{f}}{m_{p}}\right) \cdot \sqrt{m_{p}}
$$

Hence, the two simultaneous equations may be solved for either of the two unknowns to give:

$$
\begin{aligned}
& m_{p}=\left(\frac{T O F}{a}-\frac{b \cdot \Delta T O F}{a \cdot \Delta b}\right)^{2}, \\
& m_{f}=\frac{\Delta T O F}{\Delta b}\left(\frac{T O F}{a}-\frac{b \cdot \Delta T O F}{a \cdot \Delta b}\right) .
\end{aligned}
$$

At best, these equations only provide an estimate of the precursor and fragment masses (due to the poor mass accuracy of the fragment masses) and would not be sufficiently accurate for use in a database search. Instead, the estimated precursor mass is compared with a previously acquired and calibrated precursor spectrum to determine the accurate mass of the precursor. In

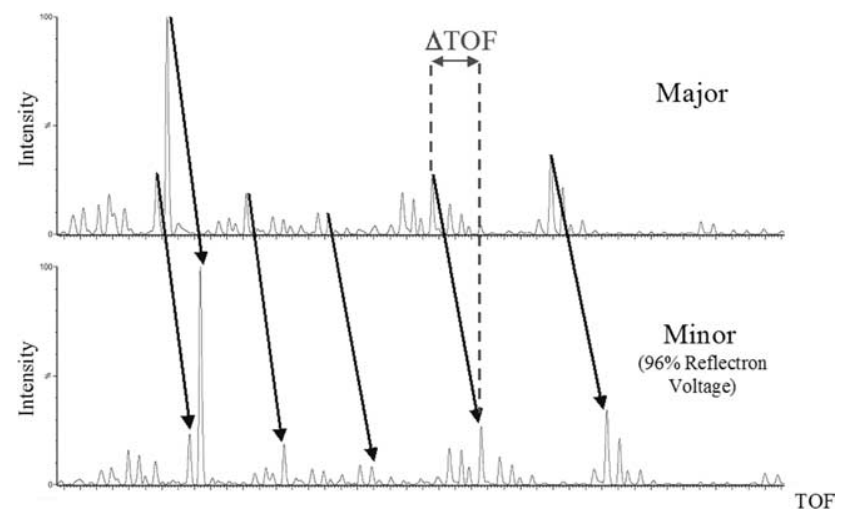

Figure 2. Typical major/minor spectra. (Top) Major spectrum, acquired at the same reflectron voltage as in a conventional PSD experiment. (Bottom) Minor spectrum which was acquired with a reflectron voltage $4 \%$ lower than that for the major spectrum.

practice, this involves matching the estimated precursor mass to the most intense precursor within a certain tolerance or search window. This matched accurate value may then be used to calculate the mass of the fragment with better accuracy. It is worth noting that this calculation can be made twice, once from the major data and once from the minor, allowing an improvement in the accuracy of the overall measurement.

Figure 2 is an example of a typical major and minor pair of spectra. The top spectrum in the figure is the major spectrum, acquired at the same reflectron voltage as in a conventional PSD experiment. The bottom spectrum is the minor spectrum which was acquired with a reflectron voltage $4 \%$ lower than that for the major spectrum. The two spectra appear very similar, and it is straightforward to match the fragment ions by eye, allowing the shift in the time of flight for each of the ions to be determined. The $\triangle T O F$ for one particular ion has been highlighted in the figure. The figure of a $4 \%$ reduction in reflectron voltage when stepping from the major to minor spectrum was found empirically and is a compromise between maximizing the accuracy of the estimated precursor estimate ion mass, with the equally important consideration that the larger the shift the smaller the region of overlap for the focused regions of the major and minor spectra. For example, assuming an inaccuracy of $100 \mathrm{ppm}$ in the TOF of a minor PSD fragment ion, with a $4 \%$ minor shift this translates to $<1 \%$ error in the estimate of the precursor mass using eq 5. Traditional timed ion gates will typically have a resolution of 100-300 and so the accuracy of the mass assignment, although not a direct like for like comparison, is comparable. Experimental confirmation of this accuracy is presented within the Results section of this article.

Matching the fragment ions by visual inspection in the manner illustrated in Figure 2 is a slow process, and can be particularly confusing if fragments from several precursor ions appear close together in time. Therefore, for this method to be practical, it has been necessary to 
Table 1. (a) and (b): Candidate fragment ion masses for major (left) peaks and minor (right) peaks. When fragment masses in (a) and (b) match they can be assigned to the relevant precursor

\begin{tabular}{|c|c|c|c|c|c|c|c|c|c|c|c|}
\hline \multirow[b]{2}{*}{$\begin{array}{c}\text { Parent Mass } \\
(\mathrm{Da})\end{array}$} & \multicolumn{5}{|c|}{ Fragment (major) } & \multirow[b]{2}{*}{$\begin{array}{c}\text { Parent Mass } \\
\text { (Da) }\end{array}$} & \multicolumn{5}{|c|}{ Fragment (minor) } \\
\hline & A & $\mathrm{B}$ & $\mathrm{C}$ & $\mathrm{D}$ & $E$ & & $A^{\prime}$ & $\mathrm{B}^{\prime}$ & $C^{\prime}$ & $\mathrm{D}^{\prime}$ & $E^{\prime}$ \\
\hline 968.48 & 766.88 & 852.45 & 942.22 & 1003.43 & 1254.32 & 968.48 & 575.34 & 683.43 & 766.88 & 843.11 & 913.14 \\
\hline 1312.68 & 852.45 & 1111.24 & 1264.62 & 1398.54 & 1573.32 & 1312.68 & 1034.35 & 1111.24 & 1275.00 & 1370.32 & 1103.34 \\
\hline 1618.84 & 942.22 & 1264.62 & 1405.51 & 1646.45 & 1896.43 & 1618.84 & 1193.34 & 1293.32 & 1332.33 & 1397.24 & 1405.48 \\
\hline 2312.15 & 1003.43 & 1398.54 & 1646.45 & 2139.29 & 2246.56 & 2312.15 & 1406.73 & 2139.29 & 2398.32 & 2497.22 & 2633.23 \\
\hline 2700.39 & 1254.32 & 1573.32 & 1896.43 & 2246.56 & 2541.94 & 2700.39 & 2287.34 & 2324.13 & 2489.33 & 2541.94 & 2800.32 \\
\hline
\end{tabular}

automate the process of matching the same fragment ions in both major and minor spectra and then calculate the precursor mass for each pair of matched peaks. As described above, this has been achieved by utilizing data from the conventional precursor mass spectrum, i.e., a previously acquired PMF.

In the matching algorithm, a user defined number of most intense precursor masses (candidate precursors) from the conventional precursor mass spectrum are used to calculate a fragment ion mass for each fragment peak in both the major and minor mass spectra. Therefore, for each fragment peak there will be as many calculated candidate fragment ion masses as there are candidate precursor ion masses. The lists of candidate fragment ion masses from the major/minor spectra are compared. A match is deemed to have been found when the difference between two calculated fragment ion masses fall within a user specified tolerance or window. This window is normally set according to the expected accuracy of mass measurement. When a match is found, the fragment ion is assigned to the precursor mass that produced the match.

A hypothetical, "perfect" example is demonstrated in Tables $1 \mathrm{a}$ and $\mathrm{b}$, which are tables of candidate fragment ion masses calculated for five candidate precursor masses for a pair of major and minor spectra respectively. For example, fragment $\mathrm{A}$ in the major data matches fragment $C^{\prime}$ in the minor data; hence these two fragments are considered to be the same and to have originated from the precursor of mass $968.48 \mathrm{Da}$. Similarly, fragment $C$ could possibly be matched to either fragment $\mathrm{A}^{\prime}$ (precursor 2312.15 Da) or $\mathrm{E}^{\prime}$ (precursor $1618.84)$. However, only $E^{\prime}$ matches within our speci-

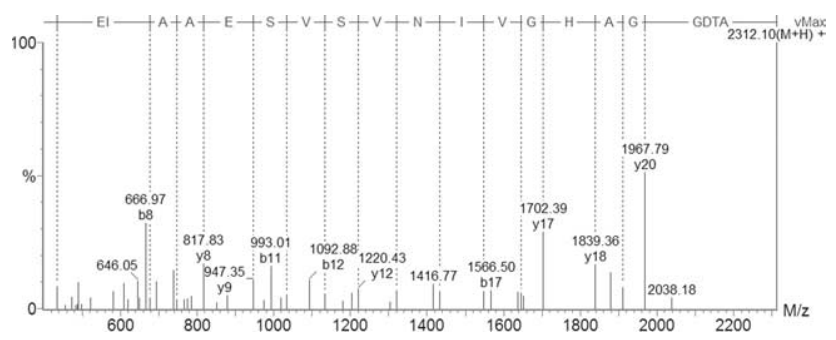

Figure 3. Typical parallel PSD spectrum of the peptide ATDGGAHGVINVSVSEAAIEASTR from a tryptic digest of alcohol dehydrogenase $(\mathrm{ADH})$ fied tolerance of $100 \mathrm{ppm}$ and so $\mathrm{E}^{\prime}$ is considered to be the matching fragment, and they both originate from the precursor of mass $1618.84 \mathrm{Da}$. In this manner, the whole peak matching and precursor assignment process is automated. The only input parameters that are required are a mass tolerance for matching fragments and precursors and the number of precursors to be considered. The length of time it takes to match all of the fragments to precursors is dependent upon the number of segments of PSD data and the complexity of the precursor/fragment spectra. Typically, the matching process for the complete set of precursor and fragment ions will take $<10 \mathrm{~s}$ to compute on a $2 \mathrm{GHz}$ Pentium 4 or equivalent PC. The final deconvoluted PSD spectra consist of centroid data rather than continuum data.

\section{Results}

Figure 3 is a typical PSD spectrum obtained using this parallel technique. Specifically it is a spectrum of the peptide ATDGGAHGVINVSVSEAAIEASTR from 500 fmol of a tryptic digest of alcohol dehydrogenase $(\mathrm{ADH})$, which has a nominal mass $[\mathrm{M}+\mathrm{H}]$ of $2312 \mathrm{Da}$. Six segments of PSD data were acquired and then stitched, in this instance giving focused coverage down to $\sim 400 \mathrm{Da}$. As can be observed in the figure, the majority of both the $b$ and $y$ ion series has been

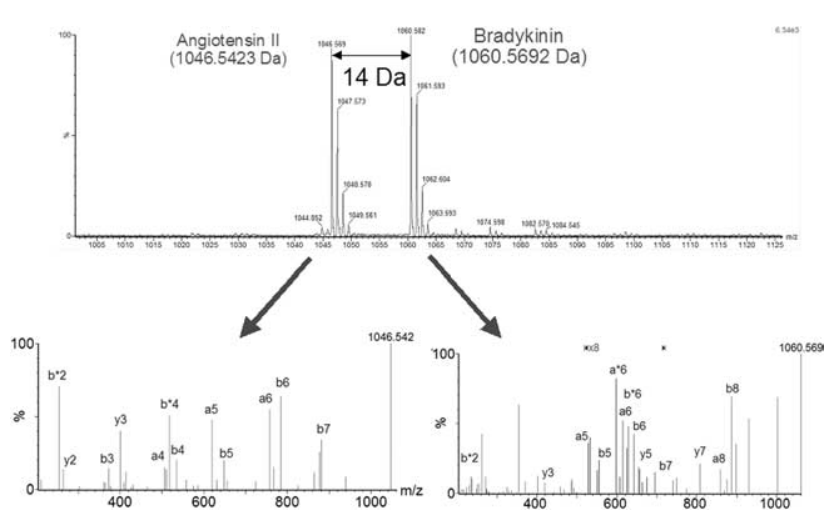

Figure 4. Top: MS spectrum of two peptides, Angiotensin II ([M $+\mathrm{H}]=1046.542 \mathrm{Da})$ and Bradykinin $([\mathrm{M}+\mathrm{H}]=1060.569 \mathrm{Da}$. Bottom Left: Parallel PSD spectrum for angiotensin II. Bottom Right: Parallel PSD spectrum for bradykinin. 


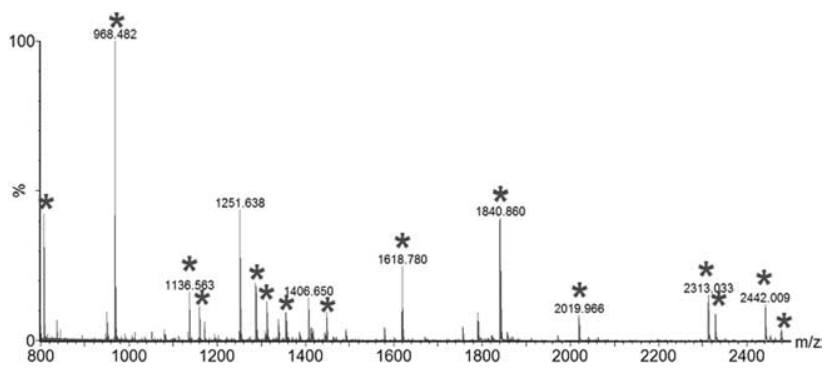

Figure 5. Precursor ion (MS) spectrum of a mixture of two standard protein digests, $\mathrm{ADH}$ and enolase.

identified, in addition to several $a$ and $z$ ions. Of particular interest when considering the PSD spectrum in Figure 3 is the low level of noise. This is an advantage resulting from the peak matching process. Not only does a noise peak, either chemical or electronic in nature, have to appear in both major and minor spectra, it also has to "shift" the correct amount to be matched to a particular precursor ion. The resulting "filtered" spectrum will be advantageous when submitting PSD data for database searching for example.

The ability of the peak matching process to differentiate fragments from precursors of similar mass is a prime consideration. To demonstrate the accuracy with which this is possible, a parallel PSD experiment was performed on a mixture of two standard peptides, angiotensin II $([\mathrm{M}+\mathrm{H}]=1046.542 \mathrm{Da})$ and bradykinin $([\mathrm{M}+\mathrm{H}]=1060.569 \mathrm{Da})$, with $250 \mathrm{fmol}$ of each peptide spotted on target. These two peptides are separated by $14 \mathrm{Da}$ and an ion gate would typically require a resolution of the order of $\sim 100$ (FWHM) to be able to isolate one precursor from the other. Figure 4 is a precursor mass spectrum of the peptide mixture accompanied by the two deconvoluted parallel PSD spectra. It can be seen that a significant number of the fragment peaks have been correctly matched to their correspond- ing precursor. Of all the peaks annotated, only one has been incorrectly assigned (marked with a asterisk). Although assigned to bradykinin, this peak is the " $y^{*} 3$ " peak from angiotensin II.

A second important consideration with this parallel PSD technique is in regard to the complexity of sample that the peak matching algorithm can handle, or stated another way, how many simultaneous PSD spectra can be acquired? To assess this, a parallel PSD experiment was performed on a 1:1 mixture of two standard protein digests, $\mathrm{ADH}$ and enolase with $100 \mathrm{fmol}$ of each protein spotted onto a single target spot. Figure 5 shows the MS spectrum obtained for this mixture.

The 20 most intense peaks from the MS spectrum were considered as candidate precursors and the data processing algorithm calculated a list of fragment masses associated for each precursor. The results were searched against the Swiss-Prot protein database using the Mascot search engine (Matrix Science, UK) integrated into ProteinLynx Global Server (Waters Corporation, Manchester, UK) and led to the identification of the two proteins (defined as an ion score of $>31$ ) in addition to a third protein, $\mathrm{ADH} 2$, an isoform of $\mathrm{ADH}$. In total 15 peptides, 8 from ADH1, 6 from enolase, and 1 from ADH2, were identified based solely upon the parallel PSD data. The Mascot identification of ADH2 contains only a single peptide, which is unique to ADH2 (nominal mass $2477 \mathrm{Da}$ ). This has a very weak ion score of 2, which isn't sufficient to confidently identify the protein. However, from data acquired previously, [both peptide mass fingerprint data and PSD data obtained on the same prototype instrument], it is known that the ADH sample does contain a mixture of the two isoforms, ADH1 and ADH2. Table 2 summarizes these results and the 15 identified peptides are highlighted in Figure 5.

It is interesting to note that the peptides yielding most fragment ions are not necessarily the most intense

Table 2. Summary of peptides simultaneously identified using parallel PSD

\begin{tabular}{|c|c|c|c|c|c|c|}
\hline Protein & $\begin{array}{c}\text { Protein } \\
\text { Score }\end{array}$ & $\begin{array}{l}\text { Measured } \\
\text { mass (Da) }\end{array}$ & $\begin{array}{l}\text { Residual } \\
\text { (Da) }\end{array}$ & $\begin{array}{l}\text { Peptide } \\
\text { score }\end{array}$ & Rank & Peptide \\
\hline \multirow[t]{8}{*}{ ADH1 YEAST } & 93 & 968.51 & 0.02 & 27 & 1 & EALDFFAR \\
\hline & & 1136.58 & 0.01 & 19 & 1 & GVIFYESHGK \\
\hline & & 1312.67 & -0.01 & 1 & 4 & SIGGEVFIDFTK \\
\hline & & 1355.61 & 0.00 & 5 & 1 & CCSDVFNQVVK \\
\hline & & 1447.76 & -0.04 & 8 & 1 & VVGLSTLPEIYEK \\
\hline & & 1618.82 & -0.02 & 9 & 1 & VLGIDGGEGKEELFR \\
\hline & & 2019.02 & -0.05 & 19 & 1 & LPLVGGHEGAGVVVGMGENVK \\
\hline & & 2312.09 & -0.05 & 6 & 1 & ATDGGAHGVINVSVSEAAIEASTR \\
\hline \multirow[t]{6}{*}{ EN01 YEAST } & 91 & 807.45 & 0.02 & 14 & 1 & TFAEALR \\
\hline & & 1159.61 & 0.00 & 28 & 1 & IGSEVYHNLK \\
\hline & & 1286.71 & 0.00 & 17 & 1 & NVNDVIAPAFVK \\
\hline & & 1840.91 & -0.02 & 9 & 1 & SIVPSGASTGVHEALEMR \\
\hline & & 2328.00 & -0.05 & 4 & 1 & IEEELGDNAVFAGENFHHGDK \\
\hline & & 2441.09 & -0.05 & 19 & 1 & IEEELGDNAVFAGENFHHGDKL \\
\hline \multirow[t]{3}{*}{ ADH2 YEAST } & 48 & 968.51 & 0.02 & 27 & 1 & EALDFFAR \\
\hline & & 2019.02 & -0.05 & 19 & 1 & LPLVGGHEGAGVVVGMGENVK \\
\hline & & 2477.11 & -0.03 & 2 & 2 & YSGVCHTDLHAWHGDWPLPTK \\
\hline
\end{tabular}


in the MS spectrum. The 1251 Da peptide, which has previously been observed to be particularly stable [14], yielded few PSD fragments and is a good example of the benefits of the parallel approach. In a conventional PSD experiment, time would likely have been spent trying to obtain PSD spectra from the most intense precursors identified in the precursor spectrum. As such, the $1251 \mathrm{Da}$ peak would have been the second precursor to have been isolated using the ion gate to acquire PSD data. Time and sample would therefore have been consumed trying to obtain data from a peptide that is not as amenable to fragmentation as other peptides within the sample.

It is interesting to compare the amount of time and sample required to acquire the data for this parallel PSD experiment with that using the conventional serial method. If PSD data had been acquired for the 15 peptides using a conventional PSD approach, it would have required the acquisition of 15 PSD spectra with six segments per spectrum; a total of 90 segments. Using the parallel PSD approach described here, just six major and six minor segments are required for a single spectrum; a total of 12 segments. Hence, the conventional serial method would have required 7.5 times more sample. This difference would be even greater if the time acquiring PSD data from unproductive peptides is taken into account. In many instances, it is unlikely that it would have been possible to acquire useful PSD spectra from 15 peptides from a single target spot using the conventional serial approach.

One limiting factor with the majority of search engines is that the precursor ion masses and the fragment ion masses must both be entered as either monoisotopic or average mass, as opposed to monoisotopic for the precursors and average mass for the fragments. As the same instrument parameters are used for all precursors, it is difficult to find a single set-up that will allow isotopes to be observed on all fragments from all precursors. It has been found to be advantageous to smooth the data so that all the fragments may be considered as average masses. However, to obtain good results from a database search, it is desirable to use the accurate monoisotopic mass of the precursor. One solution to this problem is to apply a gain and offset to the average fragment ion masses to convert them to pseudo-monoisotopic masses. The gain and offset value were obtained by plotting the monoisotopic mass against the average mass of many common peptides and fragments, then fitting a least-squares fit through the data (Figure 6a). The RMS mass error introduced by this approach is $0.03 \mathrm{Da}(<40 \mathrm{ppm})$, which is significantly lower than the $+/-0.5$ Da search tolerance commonly used with PSD data. A residual plot displaying the mass errors for the calibrant peptides is presented in Figure 6b.

In some cases, the result of the peak matching computation may be ambiguous, that is, it may not be clear which two fragment peaks are the same species. There may, for example, be a second possible solution
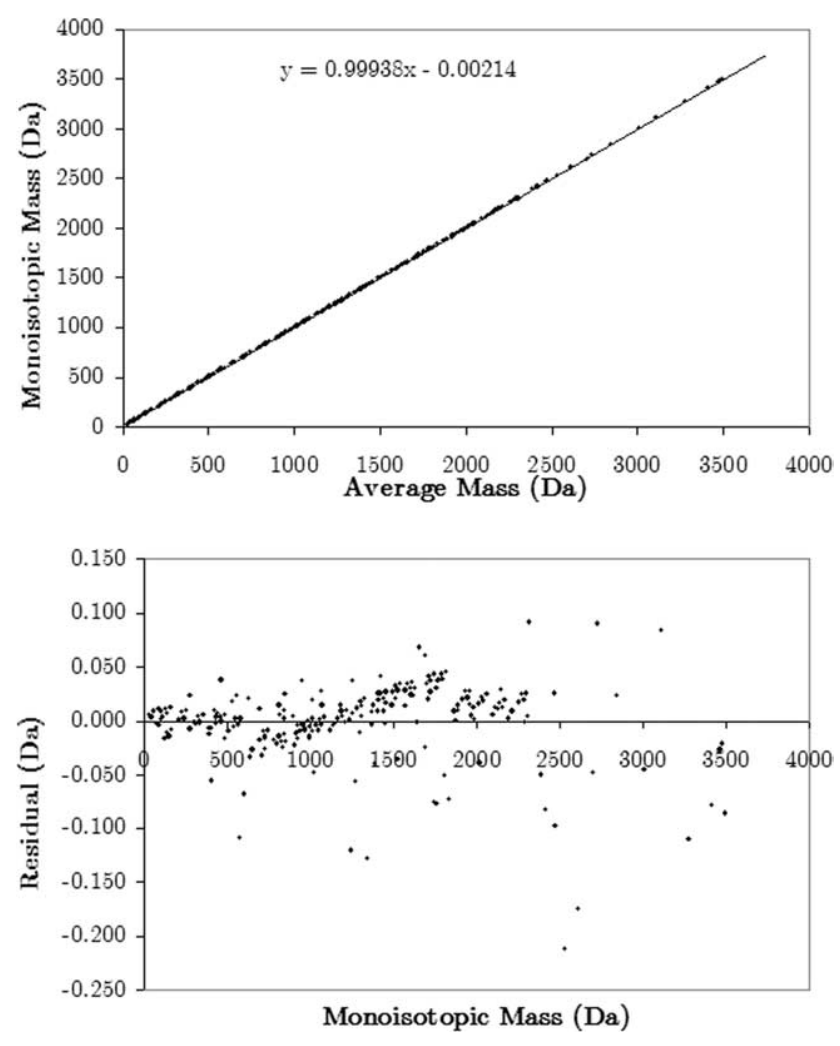

Figure 6. (a) Least-squares fit of monoisotopic mass plotted as a function of average mass for a series of common peptides. (b) Residual plot highlighting deviation of data presented in (a) from line of best fit.

for one of the fragment peaks. This ambiguity may be resolved by the acquisition of a third set of segments, i.e., a second set of minor segments. This second set of minor segments may be acquired by a further reduction of $4 \%$ of the reflectron voltage at each segment of the PSD experiment. This second set of minor segments may also be used to confirm the peak matches derived from the major and first minor set of segments.

\section{Conclusions}

We have developed a novel enhancement to MALDIPSD analysis which allows fragment ions from multiple precursor ions to be recorded simultaneously. Initial data suggest that the accuracy of precursor assignment is comparable to the resolution of a traditional ion gate and that up to 15 PSD spectra can be simultaneously acquired. The parallel experiment is easy to set up and perform and has the following advantages: (1) a reduction in the acquisition time and sample consumption in comparison to traditional PSD which scales with the number of precursor ions, (2) no decision has to be made as to which precursor ions to investigate, all precursor ions liable to fragmentation via PSD are recorded avoiding wasting time and sample investigating more stable precursor ions, (3) no ion gate is required which allows a simpler mass spectrometer to 
be constructed with increased transmission and easier operation, and (4) reduced noise levels resulting from the peak matching process.

\section{Acknowledgments}

The authors thank Richard Tyldsley, Emmanuelle Claude, Jeff Goshawk, and Martin Lunt for their help and assistance during the development of this technique.

\section{References}

1. Spengler, B.; Kirsch, D.; Kaufmann, R.; Cotter, R. J. Metastable Decay of Peptides and Proteins in Matrix-Assisted Laser-Desorption Mass Spectrometry. Rapid Commun. Mass Spectrom. 1991, 5, 198.

2. Spengler, B.; Kirsch, D.; Kaufmann, R.; Jaeger, E. Peptide Sequencing by Matrix-Assisted Laser-Desorption Mass Spectrometry. Rapid Commun. Mass Spectrom. 1992, 6, 105.

3. Spengler, B. J. Post-Source Decay Analysis in Matrix-Assisted Laser Desorption/Ionization Mass Spectrometry of Biomolecules. Mass Spectrom. 1997, 32, 1019.

4. Henzel, W. J.; Billeci, T. M.; Stults, J. T.; Wong, S. C.; Grimley, C.; Watanabe, C. Identifying Proteins from Two-Dimensional Gels by Molecular Mass Searching of Peptide Fragments in Protein Sequence Databases. Proc. Natl. Acad. Sci. U.S.A. 1993, 90, 5011.
5. Mann, M.; Hojrup, P.; Roepstorff, P. Use of Mass Spectrometric Molecular Weight Information to Identify Proteins in Sequence Databases. Biol. Mass Spectrom. 1993, 22, 338.

6. Pappin, D. J.; Hojrup, P.; Bleasby, A. J. Rapid Identification of Proteins by Peptide Mass Fingerprinting. Curr. Biol. 1993, 3, 327.

7. James, P.; Quadroni, M.; Carafoli, E.; Gonnet, G. Protein Identification by Mass Profile Fingerprinting. Biochem. Biophys. Res. Commun. 1993, 195, 58.

8. Eng, J. K.; McCormack, A. L.; Yates, J. R. An Approach to Correlate Tandem Mass Spectral Data of Peptides with Amino Acid Sequences in a Protein Database. J. Am. Soc. Mass. Spectrom. 1994, 5, 976.

9. Peng, J.; Gygi, S. P. Proteomics: The Move to Mixtures. J. Mass Spectrom. 2001, 36, 1083 .

10. Wall, D. B.; Berger, S. J.; Finch, J. W.; Cohen, S. A.; Richardson, K.; Chapman, R.; Drabble, D.; Brown, J.; Gostick, D. Continuous Sample Deposition From Reversed-Phase Liquid Chromatography to Tracks on a Matrix-Assisted Laser Desorption/Ionization Precoated Target for the Analysis of Protein Digests. Electrophoresis 2002, 23, 3193.

11. Mamyrin, B. A. Laser Assisted Reflectron Time-of-Flight Mass Spectrometry. Int. J. Mass Spectrom. Ion Processes 1994, 131, 1.

12. Cotter, R. J. Time-of-Flight Mass Spectrometry: Instrumentation and Applications in Biological Research; American Chemical Society: Washington, 1997, p. 62.

13. Bradbury, N. E.; Nielsen, R. A. Absolute Values of the Electron Mobility in Hydrogen. Phys. Rev. 1936, 49, 388.

14. Wattenberg, A.; Organ, A. J.; Schneider, K.; Tyldesley, R.; Bordoli, R.; Bateman, R. H. Sequence Dependent Fragmentation of Peptides Generated by MALDI Quadrupole Time-of-Flight (MALDI Q-TOF) Mass Spectrometry and Its Implications for Protein Identification J. Am. Soc. Mass Spectrom. 2002, 13, 772. 